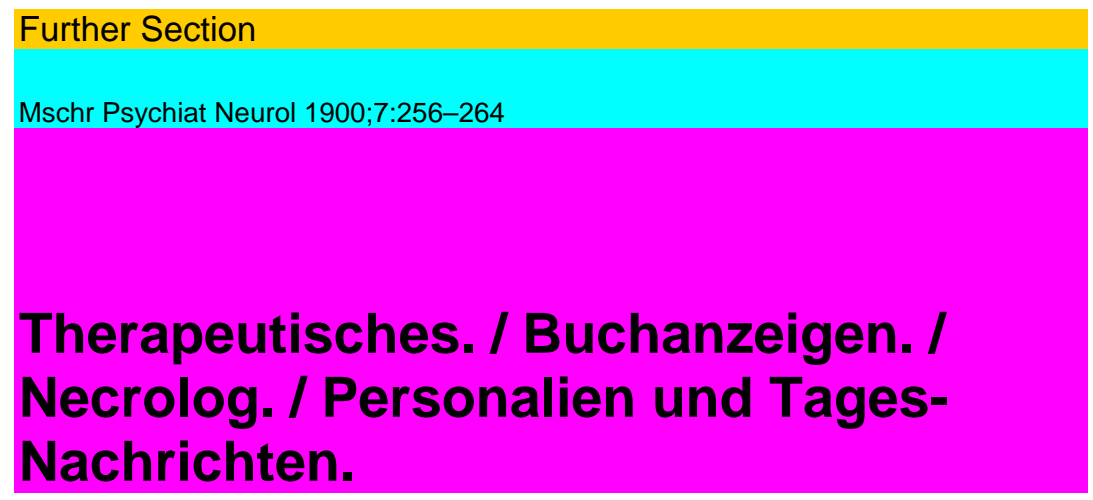

Hitchcock berichtet fiber 206 F\&lle acuter Manie (im englischen Sinn, also incl. Paranoia halluc. acuta bezw. Amentia), welche ohne Sedativa behandelt wiirden. Er verzeichnet $83 \mathrm{pCt}$. Ge-nesungen, 3,3 pCt. Todesfalle. Die durchschnittlicbe Behandlungs-dauer der Manner betrug 3\% Monate, der Frauen 5 Monate. In einzelnen Fallen wirkten Abfuhrmittel (Calomel) ausgezeichnet. Ge-legentlich wurden feuchte EiDpackungen, warme Bfider etc. verwandt. Verdiinnten Whiskey und Stout betrachtet Verf. offenbar nicht als Sedativa. In der an den Vortrag sich anschliessenden Discussion waren die Meinimgen sehr geteilt (Journ. of ment. sc. Jan. 1900).

Aus den zehn Jahresberichten des Asylums Commitee of the London County Council ergiebt sich, dass von den innerhalb der. letzten vier Jahre (1895-1898) als geheilt entlassenen Geistes-kranken 20\%ipCt. bereits bis zum 31. Marz 1899 wegen Riickfalls wieder aufgenommen werden mussten. Bei $13 \%$ pCt. trat der Riick-fall bereits im ersten Jahr nach der Entlassung ein (ibidem). 


\section{Buch anzeigen.}


H. Fischer teilt einen Pall von Gangran der Weichteile und des Knochens beider Ftisse bei einem Paralytiker mit, in welchem die von Stumpf-Werneck empfohlene Thonbehandlung (reicb.iich.es Aui'streuen von Argilla) eine glanzende Heilung der gangranosen Teile herbeifiihrte. (Munch. Mẹd. Wochenschr. 1899.)

Winternitz jun. hat in der Mering'schen PolikliDik i'estgestellt, dass unter dem Einfluss heisser Bader die Warmebildung und der Kohlenstoffumsatz sehr erhebl'ich steigt. (Klin. Jahrb. 1899.)

Aspirin, ein Derivat der Salicylsaure, welches vor der letzteren den Vorzug hat, keine MagenstOrungen hervorzurufen und besser $\mathrm{zu}$ schmecken, wird neuerdings auch gegen tabische Schmerzẹn und Neuralgien empfohlen. Die Dosis betragt $1 \mathrm{~g}$, mit Zucker in einem. Bssloffel Wasser. (Witthauer, Therap. Monatsh., 1899, Xo. 6; Weil, Allg. Med. Centralztg., 13. Januar 1900).

\section{Jiucli anzeigen.}

Obersteiner, H.: Arbeit en aus dem Institut f ii $r$ An at o mi e und Physiologie des Centralnervensystems an der "Wiener T Jniversitiit. VI. Heft. Leipzig 1899, Franz Deuticke.

Das neue Heft, mit acht Tafeln und sechs Abbildungen im Texte aus-gestattet, bietet in sech's schatzenswerten Abhandlungen viel Interessantes.

1. S S itzer, A l ex a nder: Ein Fall von Tumor am Boden der

Rautengrube. Beitrag zur Kenntnis des hinteren Liingsbundels.

Bei 34 jahrigem Tuberkulosem: anfangs Deviation der Augen und des Kopfes nach rechts, Schwindel beim Kopfdrehen nach links. Spater habi-tuelle Senkung und massige Linkswendung des Kopfes, Schwindelgefiihle, Nausea bei Erheben und Hintenwartsneigen des Kopfes. Keine Hirn drucker-soheinungen. Befund: vornehmlichlinksseitiger, soharf begrenzter, iiberhasel-nussgrosser Solitiivtuberkel im dorsalen Haubenteil. Auf- und absteigende Degenerationen. Die ausfubrlich wiedergegebenen Befunde und Unter-suchvmgen Anderer veranlassen Verf., im hinteren Langsbiindel den „moto-rischen Schenkel eines zur raumlichen Orientierung dienenden Reflexbogens" zu sehen, dessen Centren Hinterstrangskerne, Deiters'scher Kern, Mittelund Zwischenhirn darstellen und der zu den Vorderhornzellen des Hals-markes und den Augenmuskelkernen Impulse abgiebt und so mit beitragt, uns die Orientierung im Raume zu ermoglichen, dem „Aufbau unserer extensiv geordneten Vorstellungswelt" dient.

2. Zap pert, Julius: Deber Wurzel- und Zellveranderungen im Centralnervensystem des Kindes.

Zappert berichtet ttber 140 frilher und neu untersuchte kindliche Riickenmarke (auch Teile der Medulla oblongata und motorische Hirn-nerven wurden untersuebt) und kommt in der Hauptsache zu folgenden interessanten Ergebnissen: 1. Im Ruckenmark von Kindern aus den ersten zwei Jahren sind Degenerationen der intraspinalen Anteile der vorderen Wurzeln ein recht haufiger Befund, z. T. physiologische Verbaltnisse darstellend, z. T. jedenfalls krankhafte. 2, Aehnliehe Veranderungen zeigen ofters die motorischen Hirnnervenwurzein, namentlich Nn. HI, V mot., VI, XI und ebenso die von den Clarke'schen Saulen ausgebenden, zur Klein-bimseitenstrangbahn ziebenden Fasern. 3. Die hinteren Wurzeln zeigen weniger haufig und intensiv als die vorderen solche Degenerationen, in seltenen Fallen sind auch die motorischen Vorderhornzellen alteriert. 


\section{Buchanzeigen.}

3. Neurath, Budolf: Beitrag zur postinfectiosen Hemiplegie im Kindesalter und zur pathologischen Anatomie des kindlichen Centralnervensystems (Neurogliosis gangliocellalaris diffusa). S. $131 \mathrm{ff}$

Mikroskopische Untersuchungeiner bei einem an scarlatinSser Nephritis unter terminal hemiplegischen Ers'cheinungen yerstorbenen Knaben ge-i'undenen diffusen und disseminierten Hirnsklerose-. Dichtes Gliagewebe und eigenartige, Ganglienzellen ahnliche Zellen mit glasig-hyalinem Proto-plasma, mehrfach geteiltem Kern und Fortsatzen fanden sich in knorpel-harten Herden una auch diffus zerstreut in scheinbar sonst normalem Gewebe.

4. Sc huzo Kure: Die normale und pathologische Structur der Zellen

an der cerebralen Wurzel des N. trigeminus, die Kreuzungsfrage der

letzteren und der motorischen Trigeminuswurzel. S. $158 \mathrm{ff}$.

Experimentelle Untersuchungen am Kaninchen. Wesentliche Ergeb-nisse: Zellen an der cerebralen Wurzel und gewisse Zellen im Locus coeruleus haben eigenartigen Charakter (ahnlich den Spinalganglienzellen) und demgemass wohl auch bestimmte andere Function (als der motorische Kern). Die diesen Zellen zugehorigen centralen und die motorischen "Wurzelfasern kreuzen sich nicht.

5. Pineles, Friedrich: Zur Lehre von den Functionen des Klein-

hims. S. $182 \mathrm{f}$.

- Interessante im Anschluss an klinische Beobachtungen gegebene Er-Srterungen tlber die Beziehungen des Kleinhirns zur Motilitat, des Klein-hirns und der Bindearme zu Chorea- und Athetose-Bewegungen (bei halb-seitigen Paresen), in denen Pineles Ausfallserscheinungen sieht, bedingt durch Bindearm- bezw. Kleinhirnlasion.

6. Zappert, Julius und Hitschmann, Fritz: Ueber eine unge-

wohnliche Form des angeborenen Hydrocephalus. S. $225 \mathrm{ff}$.

Ausfuhrliche Bescbreibung einer Art Hydromikrocephalus, der sich bei einem 11

Tage alten, nicht auffalligen Kinde fand. Veiff. legen die Anfange der interessanten Missbildung - die Stammganglien fehlten - bis vor den dritten Intrauterinmonat zuriick.

H. Pfister (Freiburg i./Br.)

C. Moeli (Lichtenberg): Die Geistesstorungen im biirgerlichen Gesetzbuche und in der Oivilprocessordnung vom 20. Mai 1899. Berlin 1899. Aug.

Hirschwald, 47 Seiten. Moeli bespricht vom psychiatrischen Standpunkte aus die verschiede-nen geanderten Bestimmungen uber die Entmtindigung infolge Geistes-stfirung nach dem btixgerlichen Gesetzbuche und der geanderten Oivilprocessordnung, indem er die Entwickelung erwahnt, die die einzelnen

Bestimmungen wahrend der Beratung durchgemacht haben, und weist auch auf Quellen, Erklarungen und Motive aus der Literatur sowohl von juristi-schen als auch medicinischen Autoren hin.

Der erste Punkt betrifft dje Entmundigung wegen Geisteskrankheit und Geistesschwache und die Frage, ob jemand seine Angelegenheiten infolge Geisteskrankheit etc. nicht zu besorgen vermQge. Verf. bespricht den Unterschied zwischen Krankheit und Schwache und weist nach, was speciell unter letzterer Bezeichnung nicht verstanden werden soil - nam-lich nicht eine von der arztlichen Erfahrung nach Entstehung, Symptomen und Ausgang abgegrenzte Gruppe, sondern dass diese.zwei Ausdrilcke nur von der Beziehung zur Geschaftsiahigkeit ausgehend geschaffen sind - sie seien juristische Aequivalente - der zwei im Gesetze gebildeten Gruppen: der Minderjahrigen bis zu sieben Jahren (Kinder) und der von 7 bis 21 Jahren.

Der zweite Punkt betrifit die Geschaftsunfahigkeit desjenigen, der sich in einem die freie Willensb e stimmung ausschliessenden dauern-den Zustande krankhafter StSrung der Geistesthatigkeit be-flndet $(\$ 104,2)$ - dieser Paragraph kommt also nicht ftir Entmundigte, sondern ftir die Handlungen eines GeistesgestSrten in Betracht - w\&hrend $\S 105$ die Frage regelt, ob eine einzelne bestimmte Willenserkl\&rung in 
einem Zustande der Bewusstlorigkeit oder der voriibergeh enden Storung der Geistesthatigkeit abgegeben wurde, so dass die Erklarung ais bewusst, gewollt und rechtlich wirksam nicht angesehen werden kann.

Drittens wird der \& 1910 Âbs. 2 besprochen, der von der Pflegsohaft handelt, und insbesondere der letzte Absatz (3): Die Pflegsohaft darf nur mit Einwilligung des Gebreehlichen angeordnet werden, es sei denn, dass eine Verstandigung mit ihm nicht mSglich ist.

Viertens behandelt Moeli die Entmiindigung wegen Trunksucht $-\mathrm{d}$. h. eines krankhaften Zustandes der unwiderstehlichen Sucht zum dauern-den oder periodisch wiederkehrenden iibermassigen Genuss berauschender Getranke und diejenige wegen Verschwendung, beides Facta, die bisher nicht bestanden - also eine vollstandige Neuerung darstellen.

Es folgt fiinftens $\S 832$, der von der Haftpflicht derjenigen handelt, die korperlich oder geistig kranke. der Beaufsichtigung bedtirftige Individuen nicht geniigend zuriickhalten und bewachen, so dass Dritten Schaden zu-gefiigt wird. Die Ersatzpflicht tritt aber nicht ein, wenn der Beaufsichti-gende seiner Aufsichtspflicht geniigt hat oder wenn der Schaden auch bei gehoriger Aufsichtsfiihrung entstanden sein wurde. Dieser ebenfalls neue Paragraph betrifit $z$. B. die Falle, in denen ein ruhiger, unter Begleitung eines Warters ausgehender Epileptiker oder Paranoiker plstzlich ausser-halb der Anstalt mit Polizei und Fremden infolge Tobsucht resp. Erregung in Conflict gerat.

Im sechsten - letzten Abschnitt - bespricht Moeli die Bestimmungen iiber Nichtigkeit und Anfeehtbarkeit der Ehe $\S \S 1325,1331,1333,1337$ und 1569.

Die kurze Schrift, in Vortragsform abgefasst, umfasst also die haupt-sachlichsten Punkte der neuen Gesetzgebung und sei aui's warmste empfohlen. Wir konnen mit dem Verf. voll iibereinstimmen, dass wir in der neuen Fassung des Gesetzes eine grosse Vorsicht in der rechtlichen Behandlung Geisteskranker walten sehen-und in dem Bestreben, die rechtlichen Wirkungen der psychischen Leiden mSglichst abzustufen, einen Zug des Gesetzes begriissen konnen, der allgemein und insbesondere bei den diesen Kranken sich widmenden Aerzten Anerkennung finden muss.

Adolf Passow (Hannover).

Springfeld. Die Rechte und Pflichten der Dnternehmer von Privatkranken-, Privatentbindungs- und Privatirren-anstalten. (§ 30, E.-G.-O.) Berlin 1898. Richard Schoetz.

Vorliegendes Werkchen, von einem Fachmann geschi-ieben, der es versteht, trockene Gesetzesvorschriften in anziehender und leicht fasslicher Form darzubieten, ist, wie der Verfasser im Vorworte sagt, aus dem per-sonlichen Bediirfnisse heraus aus Handakten entstanden. So triigt es auch alle Vorziige des TJrsprunglichen wad Zeitgemassan an sich und bietet in der Form eines kleinen Nachschlagebuches jedem Interessenten rasche Be-lehrung. Das Buch zerfallt in einen allgemeinen und speciellen Teil und giebt im Anhange Reichs- vmd Ministerialerlasse, die sich mit dieser Materie beschaftigen, wieder. - Der allgemeine Teil giebt in fiinf Jiapiteln eine kurze historische Uebersicht iiber die Entwicklung der Rechts-verhaltnisse der Unternehmer, iiber die Erteilung der Concessionen (die gesetzlichen Voraussetzungen der Versagung und das Verfahren der Con-cessionserteilung); die Ausiibung der Concession; den Verlust der Concession: Straf- und Zwangsbefugnisse der Behorden.

Im speciellen Teile spricht der "Verf.:

1. Von den besonderen Berufspflichten der Unternehmer von Privatkranken- und Entbindungs-Anstalten.

2. Von den besonderen Berufspflichten der Unternehmer von Privat-irrenanstalten. 3. Von der Beaufsichtigung der Anstalten.

Die Lecture dieses Buches kann durch ein Referat in keiner "VVeise ersetzt werden. Wir empfehlen dasselbe angelegentlich. Es ist mit freiem, den Unternehmern wohhvollendem Geiste geschrieben, frei von jedem 
Buchanzeigen.

Bfireaukratismus. Besonders hinweisen mSchten war- auf den Abschnitt uber „besondere Berufspflichten der TJnternehmer von Privatirrenanstalten". Die einschlagigen Gesetze sind den Psychiater*: wenig gfinstig; die Frei-heit der Aut'nahmen, die im Interesse der Kranken gelegen ist, ist immer noch. zu sehr beschrankt. Zuwiderhandlungen gegen die betreffenden Vor-schriften, die angeblich Missbriiuche, wie sie durch die Aufdeckung der widerwartigen Zustande bei den Mariabergern ans Tageslicht gelangten, verhilten sollen, leider aber den ganzen Stand treffen statt der einzelnen, die gegen arztliches "Wissen und Siztliche Humanitat verstossen, werden so strenge geahndet, dass wir alien Beteiligten jenen Absatz (Seite 86 u. ff) ganz besonders $\mathrm{zu}$ eingehendem Studium empfehlen. Dasselbe liegt, wie der Verf. sehr richtig sagt, im eigensten Interesse jedes Unternehmers.

Mit einem Anhange (III. Teil) der frtihere wichtige Erlasse fiber An-lage, Ban etc.

enthalt, schliesst der Verf. seine belehrenden Ausffihrungen.

Frie dlande r (Frankfurt a. M.)

Gebhardt, Walther, Die mikrophotographische Aufnahme ge-f ilrbter Pra par ate. Miinchen 1899. Die vorliegende Abhandlung bezieht sich im wesentlichen auf die Photographie geiarbter Objecte und der- hierbei anzuwendenden Lichtfilter. bie beriicksichtigt in ausfuhrlicher Weise die Schwierigkeiten, welche der Anwendung passender Filter bei den verschiedenartig gefarbten Praparaten noch immer entgegenstehen. B.ef. kann namentlich die grossen Vorzuge des Zetnow'schen Filters, die auch in dieser Arbeit hervorgehoben werden, aus eigener Erfahrung durchaus bestatigen; fiir blaue und violette Farben, wie sie bei Praparaten aus dem Gebiete des Centralnervensystems vor-wiegend Verwendung finden, ist dieser Filter v6llig ausreichend. Auch die grossen Yorzuge der orthochromatischen PerutzPlatten bei Wiedergabe gefarbter Objecte kann Kef. nur bestatigen.

Sander (Frankfurt a. M.

Pflster, Herm., Ueber die occipitale Region und das Studium der Grosshimoberllache. Stuttgart, Ferd. Enke, 1899. Verf. hat an 175 kindlichen Gehirnen die Furchung des Occipital-lappens studiert und damit eine wesentliche Liicke in unseren ICenntnissen von der Himoberflache auszufiillen gesucht. Aus den Ergebnissen des ersten Teiles „Ueber die occipitale Region" ist folgendes hervorzuheben: Der Sulcus occipitalis anterior und Sulcus occipitalis lateralis haben sfets einen selbstandigen und typischen Verlauf. Das Zusammeafiessen zweier Furchen wird mit zunehmendem Lebensalter seltener, die Modellierung der Himoberflache ist noch langereZeit nach der Geburt Veranderungen unter-worfen und erstreckt sich weit fiber den ersten Lebensmonat, ja fiber das erste Quai'tal hinaus, anfangs vorhandene Furchungsconfluxe konnen daher noch spater durch Emporwachsen von Brficken zum Verschwinden kommen. Im zweiten Teil „Deber das Studium der Grosshirnoberflache" beschreibt der Verf. drei Gehirne mit besonders stark variierender Furchen-bildung der Occipitalgegend, bedingt durch extreme Lappenproportionen der benachbarten Teile. Er weist nach, dass diese extremen Hirntypen nichts Pathologisches darstellen, sondern nur die Ausdriicke der „Indivi-dualitat" sind, d. h. der Fahigkeit des Grosshirns, in den G-r6ssenproportionen seiner einzelnen Teile zu variieren. Diese Schwankungen in den relativen Dimensionen der einzelnen Hirnregionen sind ihrerseits auf die Gestaltung der Oberflachenfurchung von bestimmendem Einfluss. (Der Arbeit sind Zeichnungen beigegeben, welche die individuellen Vei-schiedenheiten der Oberflachenfurchung demonstrieren sollen.)

Sander (Frankfurt a. M.)

Wentz, Die Thatigke it des praktischen Arztes b ei Psychosen.

Miinchen 1899.

Das kleine Schriftchen bringt die Wiedergabe eines Vortrages des Verf. fiber den heutigen Stand der Diagnostik und Therapie der GeistesstSrungen, soweit sie fiir den praktischen Arzt Interesse haben, und soil 
wohl seinerseits dazu beitragen, die Scheu vor der Irrerianstalt, die noch immer bei zahlreichen Aerzten vorhanden ist, zu zerstreuen Sander (Frankfurt a. M.)

Schiirmayer, Der heutige Stand und die Forts chritte der Technik der Rontgen-Photograp hie. Miinchen 1899.

Behandelt den gegenwartigen Stand der R6ntgen-Photographie mit besonderer Berucksichtigung der teehnischen Hiifsmittel. In der Schlussbetrachtung warnt der Verf besonders vor einer Ueberschatzung des durch das Photogramm gewonnenen Bildes. das den ungeiibten Beobachter leieht zu Trugschltissen veranlasst. Sander (Frankfurt a. M.)

P. Daude (Berlin). Das Entmiindigungsverfahrengegen G eistes-kranke, Geistessch wa che , Verschw ender und Trunk-stichtige. Berlin 1899. H. W. Miiller. 151 Seiten.

Daude giebt in Vorliegendem eine Neubearbeitung seines vor 17 Jahren erschienenen Buches heraus, weil, wie Verf. im Vorwort aus-fiihrt, das Entmiindigungsverfahren, "syie es bisher nach der Civilprocess-ordnung vom 30 Januar 1877 geregelt war, durch das Gesetz vom 17. Mai 1898, betreffend Aenderungen der Civilprocessordnung, mannigfache Modifikationen erlitten hat, die hauptsachlich mit dem Burgerlichen Gesetz-buche zusammenhangen und namentlich darauf abzielen, die bisherigen Bestimmungen den familienrechtlichen Vorschriften des Burgerlichen G-esetzbuches anzupassen, ausserdem aber fur die der Civilprocessordnung bisher nnbekannte Entmiindigung wegen Geistesschwache und wegen Trunksucht das Verfahren zu regeln. Ausserdem sind die verschiedenen Bestimmungen, welche eingetretene Missstitnde beseitigen und besonders eine starkere Gewahr gegen Fehlspriiche bei der Entmiindigung wegen Geisteskrankheit schaffen sollen, ausfiihrlich besprochen und erklart. In dem Anhang finden sich die neuen Gesetze resp. Bestimmungen und acht MusterbeispLele fur die verschiedenen Arten der Antrage auf Ent-mtindigungen, Anfechtungsklagen, Wiederaufhebung u. s. f.

Bei der jetzigen Hochflut von Monographien und Abhandlungen, die

diesen Punkt betreffen, ist das vorliegende Buch aus der Feder des bekannten Berliner Juristen nur mit Freuden zu begrussen. Es sei darum aufs warmste empfohlen.

Adolf Passow (Hannover)

\section{Zulu Andenken an Ludwig Meyer.}

Am 8. Februar ist in Ludwig Meyer einer von den Mttnnern heim-gegangen, welche in DeutschlaDd den Aufbau der modernen Psychi-atrie in wissenschaftlicher und praktischer Beziehung bewerkstelligt haben. $\mathrm{Br}$ war es, welcher die pathologisch-anatoraische Porschungs-methode und Denkweise wahrend der zweiten Halfte der funfziger Jahre in die Psychiatrie eingefiibrt hat, eine Methode, die dann unter der werkthatigen Mitarbeit Westphal's, Gudden's und Meynert's die atiologische und klinisch - symptomatologische Porschungsmethode Griesinger's in fruchtbringendster Weise er-ganzte. Seine Arbeiten uber die progressive Paralyse stellen den Ausgangspunkt fiir alle spateren methodischen Porschungen auf diesein Gebiete dar. In seinen craniologischen Studien hat Ludwig Meyer es verstanden, die Arbeit des Anatomen mit derjenigen des Ethnographen und Biologen in feinsinnigster Weise zu vereinigen. Von seinen klinischen Abhandlungen mOchte ich hier nur die Studie iiber das circulare Irresein hervorneben, welche ein Meisterstuck $\mathrm{ab}$-gerundeter plastischer Darstellung ist. 
Necrolog.

Was ihn aber weit iiber den engeren Kreis der wissenschaftlichen Gemeinde emporbebt, ist seine praktische Wirksamkeit als Reformator unseresdeutschen Irrenwesens. Bs widerstrebt mir, auf die. unerquicklichen, mit Leidenschaft und Verbitterung- geffibrten Kampfe einzugehen, welehe die psychiatrischen Reformer der ftinfziger und secbziger Jabre mit iiberlebten Anscbauungen in arztlichen Facb-kreisen, mit Vorurteilen und libel angebrachter Sparsamkeit der VerwaltungsbehSrden auszni'echten batten. Diese Kampfe besitzen heute nur nocb bistorisches Interesse, da die von Ludwig Meyer und seinen. Mitkampfern vertretenen Anscbauungen, welehe aus der wissen-gchaftlich vertieften und gereinigten Erkenntnis der GeistesstSrungen beraus die Bebandlung der Geisteskranken von Grund auf umge-stalteten, gegenw\&rtig allgemeine Geltung gewonnen haben. Sie mussten aber wenigstens erwahnt werden, um der Summe von rast-loser, trotz aller Anfeindungen und Widerstande unbeirrter Arbeit aucb bei dem jiingeren Gescblecbte die verdiente Anerkennung zu verschaffen.

Icb will micb mit diesen kurzen Bemerkungen iiber die reicbe Lebensarbeit meines dabingegangenen Lehrers begniigen. Es wird an anderer Stelle von berufener Feder sein Lebensbild ausfiibrlich gezeicbnet werden. Wohl aber ist es mir ein inneres Bedtirfnis, mit wenigen Strichen die PersOnlichkeit des Mannes zu zeichnen, welcher mich vor 23 Jahren in die Psycbiatrie eingefiibrt bat. L. Meyer stand damals auf der HOhe seiner Scbaffenskraft. Die Erinnerungen an die machtvolle, harmonische Personlicbkeit sind in meiner Seele durch das Gefubl tiefster Dankbarkeit fest verankert. Icb sebe ihn im Geiste vor mir; den grossen breitschultrigen Mann mit dem machtigen Kopf, den ernsten, ausdrucksvollen Ziigen, dem giitigen, klaren Blick! Seine Haltung, sein Gang, seine Bewegungen und seine Spracbe batten etwas Gemessenes, fast Feierliches, so dass wir Jiingeren mit einer gewissen Scheu und Ehrfurcbt ihm entgegen-traten. Die Befangenheit wicb erst, nacbdem man einen BUck in das tiefe, reicbe Gemiit des Mannes getban batte, dem nichts Mensch-licbes fremd war. Jedem, dem es vergonnt war, unter seiner Leitung im Anstaltsdienste und in der wissenscbaftlicben Arbeit tbatig zu sein, trat vSllig in den Bannkreis seiner macbtyollen Personlichkeit. Ein mit der umfassendsten Allgemeinbildung vereinigtes tiefgrundiges arztlicbes Wissen und das lebendigste Interesse an alien wirtschaft-licb.en und politiscben Pragen, welcbe die Zeit beherrscbten, ver-banden sicb mit einem ausgesprocbenen Drang der Mitteilung. Auf den tSglicben Conferenzen und den gemeinsamen Anstaltsvisiten bot er uns Jiingeren aus dem unerscbopflicben Schatze seiner praktiscben Erfabrung die reicbste Belehrung, bespracb die wissenscbaftlicben Probleme, stellte uns Aufgaben und lebrte uns vor allem die engen Zusammenhange psycbiscberund somatiscberKrankbeitaerscheinungen, die Wechselwirkung zwiscben peripberer und centraler Nerventhatig-keit in jedem einzelnen Krankbeitsfalle aufsuchen. Am liebsten ver-weilte er bei seinen patbologiscb-anatomiscben und craniologiscben Studien. Die Belebrung, die wir am Sectionstiscbe von ihm empflngen, war fur uns der Anstoss $\mathrm{zu}$ eigener anatomiscber Arb\&om. Nie werde icb es vergessen, mit welcber Geduld und Nachsicht er die mikro-skopischen Arbeiten priifte und immer bereit war, belfend einzu-greifen, wenn das eigene Urteil und die eigene Erfahrung zur Deutung eines Befundes nicht ausr ${ }^{\wedge}$ mte. Er war uns alien ein leucbtendes Vorbild im Umgange mit Kranken und in der Leitung der Ver- 
waltungsgesch\&fte. Wenn L. Meyer iui angeregten Gesprache auf der Visite dureh die Krankenraume schritt, so konnte der Uneingeweihte glauben, dass er am Einzelnen achtlos voriiberginge. Dabẹi schweifte aber sein Auge uberall umher. Mit einem untriiglichen Blicke bemerkte er jede Veranderung in dem iiusseren Gebahren eines Kranken, unterbrach sein Gesprach und erorterte den Pall im Krankenexamen, erlftuterte die eingetretene Zustandsanderung und gab knappe und klare Anordnungen iiber die therapeutischen Massregeln. Das Pflegepersonal verehrte in L. Meyer den strengen, aber gerechten und wohlwollenden Herrn, welcher fortdauernd bemfiht war, die materielle Lage und sociale Stellung des Pflegepersonals zu heben. Sein Verkehr mit dem Letzteren war geradezu vorbildlich in der ruhigen, bestimmten, leidenschaftslosen Art der Zurechtweisung. Ich habe L. Meyer nur ein einziges Mai zornig gesehen und zwar in einem heftigen und gerechten Zorn, als ein Warter einen Kranken misshandelt hatte. Es fehlte nieht viel, so hatte der leidenschaftlich erregte Mann den trotzig und frech dastehenden Warter eigenhandig zur Thiire hinaus befSrdert; der Mensch retirierte indessen schon von selbst in grosster Eile. Es war eine aufregende Scene, die L. Meyer sichtlich angegriffen hatte. Die nachhaltige Wirkung auf das gesamte Wartepersonal war aber unverkennbar.

Ich fiige noch einige Bemerkungen iiber Lu.dwig Meyer als Lehrer hinzu.

Noch jetzt bewahre ich kurze Aufzeichnungen aus seinem theoretischen Colleg der PsycMatrie. Ich habe noch oft in spateren Jahren darin gelesen und war jedesmal aufs Neue von der Piille treffender Bemerkungen fiber Systematik und Nosologie angezogen. Alle jene Schwierigkeiten, denen wir noch heute nach beiden Eichtungen hin auf Schritt und Tritt begegnen, hat er in klarer und bestimmter Weise dargelegt und uns gelehrt, die alte psychologisch-klinische Methode nur dann zu verlassen, wenn neue gesicherte That-sachen eine Wandelung des Krankheitsbegriffs und eine (Jm-schmelzung der Krankheitsbilder rechtfertigten. Er war dabei der Ueberzeugung, dass ein. solcher Portschritt durch die klinisch-descriptive Methode allein nicht bewirkt werde. Er sah voraus-schauend den Tag kommen, an dem uns die neueren Methoden der atiologischen Porschung, die histochemischen und anderweitigen Untersuchungen, sowie die verfeinerte mikroskopische Technik einen tieferen Einblick in die Entstehungsbedingungen und in die patho-logischen Vorgange bei den Psychosen eroffnen wtirden.

In der Klinik untersuchte er die Palle vor den Zuhorern in sorgfaltigster Weise und knupfte an die einzelne Beobachtung abgerundete und formvollendete Vortrage, in welchen er den aktuellen Stand des behandelten Themas erschSpfend erklarte. Der Horer wurde so in die Lage gesetzt, im Anschluss an das einzelne, sich seinem Ge-dachtnis fest einpragende klinische Beispiel seinem Wissenskreise die ganze zugeh5rige Kette klinischer Einzelthatsachen anzugliedern. Dauernd ist mir in der Erinnerung geblieben das Krankheitsbild eines epileptischen Schulmeisters, welcher wahrend eines protrahierten Dammerzustandes der Klinik vorgestellt wurde. L. Meyer trug bei dieser Gelegenheit alle verschiedenen Abstufungen der epileptischen Bewusstseinsetorungen seinen HSrern vor und flocht dann andere Beispiele in die Schilderung ein, die er mit wenigen, scharfen Umrissen skizzierte. Er entwarf in dieser Weise seinen HOrern ein abgeschlossenes Gemalde des psychisch-epileptischen Krankheitszu- 
standes und war daW bemtiht, ihnen die praktische, insbesondere die forensische, Bedeutsamkeit di'eser Krankheitszustande vor Augen zu fiihren.

Die allgemeine Liebe und" Verehrung, welche L. Meyer bei seinen Sckiilern im Laufe seiner 34jahrigen akademischen Wirksam-keit sick erworben katte, gab sick in schonster Weise kund an den beiden Festtagen zur Feier seines 25jakrigen Professorenjubilaums im Jakre 1891 und an seinem 70. Gebtirtstage am 27. December 1897, den Pesttagen, welche er im Kreise seiner Pamilie, seiner Preunde und seiner Sckiiler beging. Ick war zum letzten Male bei dieser Geburtstagsfeier mit dem verekrten Manne in seinem gast-lichen Heim, in seinem Pamilienkreise zusammen und frischten wir alte Erinnerungen auf. Ick land L. Meyer sehr gealtert, die machtige Gestalt von der Last der Jahre gebeugt, jedock die geistige Kraft ungebrochen. Welch' liohen Genuss bereitete es mir, am Vorabend der Geburtstagsfeier in langem, vertrauten Gesprftche ihn aus alten Tagen erzaklen zu horen. Am liebsten verweilte er in seinen Erinnerungen bei dera Ausgangspunkte seiner Lebensarbeit, den rauk-seligen, entbehrungsvolien Tagen in der Charite, in welchen ein er-frischender, lebendiger Zug gelauterter naturwissenschaftlicher Er-kenntnis die Medicin erfiillte und eine froke, arbeitsfreudige Scbar junger Gesellen sick im eifrigsten Wettbewerbe in der wissensckaftlicken Arena tummelte. Nie ist mir das Meminisse juvat so Mar zum Bewusstsein gekommen als in diesen Stunden der Eucksckau.

Zum letzten Male sah ich L. Meyer im vorigen Soinmer an den Gestaden des Bodensees. Ein Sckweizer Perienaufentkalt hatte iko

erfrisckt und gestarkt. Wir konnten nur fliichtige Grusse tauschen,

da er heimwarts eilte. In diesem Winter vermehrten sick seine Leiden, sodass der Tod als befreiende ErlOsung gelten musste. Ehre

seinem Andenken! Binswanger (Jena).

Personalien und Tages-Nachricliten.

Dr. Mondino ist zum ordentliclien Professor der Psychiatrie in Pavia ernarmt.

Zum Direktor des stiidtischen psychiatrischen Krankenhauses in Odessa ist der Professor der Psychiatrie an der Oharkower Universitat, Dr. J. Orsehanski, gewahlt worden.

Nach einer Mitteilung des Berl. Tagebl. sind Bestrebungen im Gang, die $D$ nf all $V$ ersi ch erungspf licht auf diejenigen Greisteskranken aus-zudelmen, die in Anstalten regelmâssigmit versioherungspflichtigen Arbeiten beschaftigt werden. auf das Wartepersonal der Anstalten angeregt, welche bekanntlich bereits 1896 auf dem deutschen Trrenarztetag zu Heidelberg als wunschens-wert bezeichnet wurde. Bis jetzt steht ein Anspruch- auf dauernde Flir-sorge nur dem in Beamtenstellung befindlichen Personal der Staatsanstalt zu

Prof. Sgobbo giebt vom 1. Okt. 1899 eine neue Zeitschrift unter dem Titel Griornale di Elettricita- medica heraus. Der Jahrgang umfasst 6 Hefte und kostet 4 Lire.

Ein internationaler Congress fur medicinis.ehe Electrologie und B,adiologie wird in Paris vom 27. Juli. bis 1 . August 1900 tagen. An-meldungen und Anfragen sind an Prof. D. o u m e r, Lille, rue Nicolas Leblanc, zu richten. 